\title{
Digital Gaming, Sport and Gender
}

\author{
GARRY CRAWFORD \\ Sports Division, School of Sport and Leisure Management, Collegiate Campus, Sheffield Hallam \\ University, Sheffield, South Yorkshire, S10 2BP, UK, (E-mail: g.crawford@shu.ac.uk)
}

(Received March 2004; revised June 2004; accepted August 2004)

\begin{abstract}
This paper has developed out of ongoing research into the associated patterns of sport and digital gaming interests and participation. Specifically, this paper presents the major findings of a questionnaire based survey distributed to undergraduate students at Sheffield's two universities, and a number of follow up interviews. Contrary to popular perceptions, this research finds no evidence to suggest that participating in digital gaming can have a negative effect on levels of sports participation. Moreover, the paper argues that, for some, digital gaming may increase their interest and knowledge of sport, and can prove for many a popular source of conversation that can cross-cut and inform conversations on sport. However, contrary to many previous studies, this research indicates that there continues to be distinct gender differences in gaming patterns.
\end{abstract}

\section{Introduction}

Since the advent of the first computer game in the 1960s, and the rise of home based video gaming in the 1970 s and 1980 s, digital gaming ${ }^{1}$ has rapidly risen to constitute a major worldwide cultural industry. Today, more digital games are sold in the US and UK than books (Bryce and Rutter, 2001) and leisure software sales in the UK outstrip those of both cinema box office takings and VHS/DVD rentals (ELSPA, 2003). In the UK, leisure software sales in 2002 exceeded one billion pounds sterling, making this the largest games market in Europe and the third (to the US and Japan) largest in the world (ELSPA, 2003). Digital gaming is an immensely popular form of entertainment. Fromme's (2003) study of over a thousand school children suggested that only $2.2 \%$ of these had never played a digital game, while 56 percent of boys and $29 \%$ of girls claimed to 'regularly' play digital games. However, digital gaming is not just restricted to adolescents, with the US based Interactive Digital Software Association (IDSA 2002) suggesting that 55\% of regular console gamers and $60 \%$ of computer gamers are over the age of 35 .

There is a strong, and to some extent reciprocal, relationship between sport and digital gaming. It is evident that the first home based video game, Pong (released in the mid-70s), was loosely based upon table tennis (or 'ping-pong'). Since then, sport has continued to be a popular subject for digital gaming, with sport related games constituting the best selling genre of video (home based console) games (IDSA, 2002). Almost every sport - and major sporting competition - now has its digital equivalent, with the popularity of these games leading some journalists and 
academics to assert that there is probably now more 'sport' played by children (and probably adults too) via digital games than in fields, parks or gymnasiums. As O'Connor wrote in The Times newspaper in respect of football (soccer) related digital games:

The rate at which these games are flying off the shelves would suggest more football is being played on home computers than local fields. Which raises the question, could it be possible that virtual football is even bigger than the real thing? (O'Connor, 2002: 2)

The popularity of sport-related digital gaming (as with many other aspects of this pastime) has raised some concerns with parents, politicians, teachers, and media groups. Digital gaming has often been associated with lack of social interaction and isolation, suggesting that gamers 'retreat into...[a] fantasy world' (Miller, 1993: 2) and away from their 'real' lives. Likewise, digital gaming has often been linked with creating a generation of 'coach potatoes', who sit glued to their video consoles rather than participating in more 'sociable' activities such as playing sport. However, the necessarily isolating nature of digital gaming has been questioned by many; in particular Bryce and Rutter (2001) suggest that digital gaming can prove a very 'sociable' activity, with gamers frequently coming together via Internet and LAN connections, at gaming conventions and competition, and in bedrooms and living rooms around the world. Moreover, Fromme's survey of 1111 German school children suggested that there was no evidence to support the assertion that participation in digital gaming reduces a child's participation in sport (Fromme, 2003). On the contrary, Fromme suggested that the survey had produced some evidence (though this was not statistically significant) to suggest that 'daily use' of digital games was positively associated with increased levels of sport participation.

In addition, digital gaming is increasingly proving an extremely profitable partner for many professional sports. Both digital software and hardware manufacturers frequently advertise their products on hoardings and billboards at and around sport venues, and games companies have in the past operated as major sponsors of sport teams and events, such as Sega's endorsement of the UEFA Champions League in 1997 and their shirt sponsorship of Arsenal, Sampdoria and St Etienne football clubs in 1999/2000 (Poole, 2000). Individual sport teams and athletes can also make (often quite large) sums of money from lending their names and 'corporate identities' to digital games, and in particular in the US digital games is now only second to replica shirts in generating income from merchandising for the NFL (O'Connor, 2002).

However, digital gaming has often been viewed, in both the media and academia, as a traditionally male preserve (Cassell and Jenkins, 2000). Research, particularly in the 1980s and 1990s, consistently suggested that digital gaming was an activity dominated by male participation (see Colwell et al., 1995; Buchanan and Funk, 1996). Authors such as Cassell and Jenkins (2000) and Bryce and Rutter (2003b) offer several possible explanations as to why women have traditionally been marginalised within digital gaming. First, it is evident that women are (and continue to be) much more restricted in their leisure choices and opportunities than men (Deem, 1982, 1986; Shaw, 1994; Wearing, 1998). This is due to numerous social factors, such as constraints placed upon women's presence in 'public' places, economic constraints, domestic and caring responsibilities, the limited 'leisure' time of many women, and social expectations of women's location and 
roles within society. Second, women often lack equal access to technology. Numerous studies (such as Kielser et al., 1985) suggest that boys dominate computer technology at school, and into later life computers are often viewed as male work and leisure tools and are hence frequently 'unpopular' with women (Greenfield, 1996). Third, it is argued that the themes and goals of digital games do not reflect the interests of many women. Kinder (1991) suggests that digital games are designed primarily (if not solely) for male audiences as they are built and created around 'male' preferences and interests. Bryce and Rutter (2003a, 2003b) suggest that games often involve 'male' themes, such as violent and sport related contents, and tend to lack female characters - and when these are present they are usually portrayed in sexualised or passive roles (Greenfield, 1996; Kafai, 1996; Dietz, 1998; Bryce and Rutter, 2002). As Cassell and Jenkins write:

...video games provide a prime example of the social construction of gender. Women rarely appear in them, except as damsels requiring rescue, or rewards for successful completion of the mission. (Cassell and Jenkins, 2000: 7)

However, it has been suggested that the number of female digital gamers is increasing, as women are quickly making inroads into this once male-dominated realm, and as both a consequence and contributing factor, the nature and contents of digital games are likewise changing (Cassell and Jenkins, 2000).

Bryce and Rutter (2003a) suggest that much of the research and writing published on gender and digital gaming was written in the 1980s and 90s, and was often based upon stereotypical views of digital gamers as primarily (if not exclusively) adolescent boys, and is often, therefore, both outdated and biased in its views on digital gaming. Bryce and Rutter suggest that even within the past few years, technological and cultural changes in the nature of digital gaming means that the themes and contents of many digital games are less gendered, with more featuring female protagonists and less 'male' dominated themes. Furthermore, they suggest that leisure generally has become 'less gendered', with many other traditionally male pastimes, such as rugby and football, opening up for many women (Bryce and Rutter, 2003a; 9). There are several studies that suggest that women now constitute a considerable proportion of game players and purchasers, such as Colwell and Payne (2000), who suggest in their survey of 204 school children that $87.6 \%$ of girls compared with $96.7 \%$ of boys played digital games. However, the majority of empirical studies on gender and gaming practices (such as Funk, 1993; Kafai, 1996; Roe and Muijus, 1998; Littleton et al., 1998; Colwell and Payne, 2000) are largely (if not solely) based on surveys of school children, and thus may not necessarily reflect wider participation patterns.

This paper seeks to address these issues. It has been developed out of wider research into the relationship between sport and digital gaming. Specifically, the paper draws on data gathered from a questionnaire-based survey administered to undergraduate students in Sheffield (UK) and a number of face-to-face follow-up interviews. The aim of the questionnaire and subsequent interviews was to consider the relationship between sporting and digital gaming interests and patterns of participation. As such, it has built on the work of Fromme (2003), whose research found no discernable difference between patterns of sport participation between children who did, or did not, regularly play digital games. This research therefore 
sought to build upon previous studies and consider whether interest in sport and sport related digital games could be seen in anyway to encourage interest and/or participation in the other. Associated with this overall aim has been an addition research focus on the impact of gender on participation.

\section{Research}

The data drawn on in this paper were gathered from a questionnaire-based survey and follow-up interviews. The questionnaire was distributed at the end of 2003 and beginning of 2004, to 392 undergraduate students studying sociology, social work and cultural studies at Sheffield's two universities. The questionnaire consisted of thirty short questions, which were mainly closed-ended, offering respondents a number of 'tick-box' responses. Additionally, several questions left space to encourage respondents to provide further information (in an 'open-ended' manner). The questionnaire had two main parts. The first dealt with the respondents' levels of sport participation and sporting interests, while the second half related specifically to their digital gaming patterns. The questionnaire was then followed up via sixteen (nine male and seven female) face-to-face interviews with students selected primarily on the basis of their interest and participation in digital gaming and their willingness to participate in a follow up interview.

Relying solely on a sample of undergraduate social science students does provide a skewed sample in respect of age, gender and social class. In particular, though the age of respondents in the questionnaire sample group does range from 18 to $55,90.7 \%$ of the respondents were between the ages of 18 and 21 . Hence, it is questionable whether the results gathered here are necessarily representative of a general adult population. However, as there exists little empirical data on adult digital gamers, this survey contributes to our understanding of contemporary gaming practices and provides a basis for further research.

The skewed demographics of the sample did prove beneficial in obtaining an almost equal gender balance in respondents who had recently played digital games. Due to social science degrees in Britain attracting a higher proportion of female students than many other courses, $63.5 \%$ (of the total 392) respondents to this questionnaire were female. However, significantly fewer (only 50.2\% of female respondents, compared with $93 \%$ of male respondents) indicated that they had played a digital game 'in the last three months'. Respondents who indicated that they had not played a digital game 'in the last three months', were not required to answer any questions relating to their digital gaming patterns. Hence, the gendered skew in patterns of gaming observed in this data set provides a sample of 133 men (from an overall sample of 143) and 124 women (from an overall sample of 249) who had recently played digital games. It is this sub-set of 257 respondents that is primarily drawn on in this paper, and unless stated otherwise, all percentages presented here are taken from this sample of 257 individuals.

\section{Digital gaming and sporting interests}

As with Fromme (2003), this research found no evidence to support the thesis that digital gaming negatively affects patterns of sport participation. There are some 
slight (positive) variations in patterns of digital gaming when these are divided on the basis of those who indicated that they either did or did not regularly participate in sport, but these are too small for there to be any real statistical significance $(P>0.05)$. There is also no significant relationship between either the frequency with which an individual plays sport and their tendency to play a digital game, nor the frequency with which they play digital games and their propensity to play sport. For instance, when respondents were coded ${ }^{2}$ into those who play sport more than once a week and those who play sport less than once a week, those who play sport more than once a week are slightly less likely $(67.7 \%)$ to have recently (in the last 3 months) played a digital game than those who play sport less than once a week $(71.1 \%)$. But this is not statistically significant $\left(n=303 ; \chi^{2}=0.371 ; P>0.05\right)$. However, when respondents are $\operatorname{coded}^{3}$ on the basis of how frequently they play digital games, those who play these more than once a week appear slightly more likely to have recently (in the last month) played sport (86.0\%), compared with those who play digital games less than once a week (77.3\%). Again this is not statistically significant $\left(n=254 ; \chi^{2}=3.145 ; P>0.05\right)$.

There does appear to be a significant relationship within this data set between the frequency with which respondents play sport and their propensity to have recently played a sport related digital game. Nearly $80 \%$ of those who played sport once a week or more had recently played a sport related digital game, compared with only $49.1 \%$ of those who played sport less than once a week $\left(n=254 ; \chi^{2}=\right.$ 16.804; $P<0.001)$. This was also supported by data obtained via the follow up interviews, where of the fourteen interviewees who regularly participated in sport, eight (seven men and one woman) suggested that their interest in sport had encouraged them to play an associated sport related digital game, as indicated by 'Simon' (male, aged 20):

Yeah, I play FIFA and Pro Evo Soccer and sometimes Champ Man on me mates PC [all soccer related games], but that's about it really. I'd say, first and foremost I'm into me footy. Like, I play footy and that's the main sport I follow, so the few [digital] games I play, like when I play 'em, are just like a reflection of that...you know, 'cause that's what I'm interested in.

Hence, these data suggest that an interest and participation in sport can (for some) generate an interest in digital versions of the sport. Of the 257 respondents who indicated that they had recently played a digital game, $42.9 \%$ indicated that their participation in a sport had encouraged them to play a digital version of it. Likewise, $47.2 \%$ said that their interest in sport as a fan had encouraged them to play a related digital game. However, there were notable gender differences here. Although $52.1 \%$ of men $(n=117)$ suggested that participating in a particular sport increased their interest in an associated digital game, only $18.2 \%$ of women $(n=44)$ suggested likewise $\left(\chi^{2}=15.053 ; P<0.001\right)$. Similarly, although $57.3 \%$ of male respondents $(n=117)$ indicated that their interest in sport as fans increased their interest in sport related games based on the sport(s) they followed, only $11.8 \%$ of women $(n=44)$ suggested this $\left(\chi^{2}=17.385 ; P<0.001\right)$.

There is also evidence to suggest that digital gaming can increase an individual's interest and knowledge of sport as a fan. One third of those who answered this question $(n=160)$ suggested that digital gaming had increased their interest in following sport, and more notably, 62.7\% $(n=161)$ stated that digital games had 
increased their knowledge of certain sports. This was supported in data gathered from the follow up interviews, particularly in relation to 'foreign' sports or sports teams. An example of this is the comment made by 'Mark' (male, aged 21) who stated 'Yeah, it [digital gaming] has increased my knowledge of basketball and American sports loads'. Likewise, of the five interviewees who indicated that they played sport managements games (most commonly Championship Manager), all suggested that this had significantly increased their knowledge of sport:

You get to know loads from playing Champ Man, like names of footballers in obscure foreign teams, but like important stuff as well, like who are good international players and about footy all around the world. ('John', male, aged 21)

However, these patterns appear most notable for male rather than female respondents, with $41.4 \%$ of men (compared with only $11.4 \%$ of women) indicating that a digital game had increased their interest in following a sport $\left(\chi^{2}=12.974 ; P\right.$ $<0.001)$. Similarly, $67.5 \%$ of men $(n=117)$ suggested that a digital game had increased their knowledge of sport, while this figure was only $50 \%$ for women $(n=$ 44; $\left.\chi^{2}=4.199 ; P<0.05\right)$.

Digital gaming appeared to be a popular source of conversation for many respondents. Overall, $83.5 \%$ of respondents $(n=257)$ indicated that digital gaming was something that they would discuss with their friends, while $57.5 \%$ suggested this was something they would discuss with family members. For some, this appeared to be quite a common and regular source of conversation, with $40.5 \%$ of respondents indicating that they would usually discuss digital games with their friends at least once a week, and $15.5 \%$ suggesting that they would likewise talk about this at least once a week with family members. In particular, data gathered from the follow up interviews suggested that it was sport related games that constituted the most discussed type of game. This was most notable in conversations between male friends, as all of the male interviewees indicated that conversations about sport related games would often be drawn from and inform wider conversations relating to sport. One such example was recounted by 'Mark' (male, aged 23):

...Like Championship Manager is probably the most talked about...[game] we do and...there's not a point in the day where... it'll be referenced somewhere in my day and me and my mate Tony always talk about it... and we'll talk about real football as well, 'cause they're pretty much the same thing aren't they.

These patterns were most notable for male questionnaire respondents, with only $20.4 \%$ female respondents $(n=124)$ suggesting that they would discuss digital games at least once a week with friends compared with $59.1 \%$ of male respondents $(n=133)$.

Overall, the data collected from this research reinforce the assertion of Bryce and Rutter (2001) that digital gaming is not necessarily an individual and isolating activity, but rather can be quite a 'sociable' activity. For instance, $28 \%$ of respondents suggested that they would play digital games with other people (in person) either 'every time' or 'most of the time', with only $22.2 \%$ indicating that they always played digital games alone (see Table 1). However, few respondents indicated that they would play games with others over the Internet (see Table 2).

However, these patterns of play were divided on the basis of gender, with $40.6 \%$ of the 133 male respondents suggesting that they played digital games 'every time' 
Table 1 Percentages of how frequently respondents play digital games with other people in person (number) $(n=254)$

\begin{tabular}{lrrr}
\hline Play with others 'in person' & Overall & Male & Female \\
\hline Every time & $9.7(25)$ & $14.3(19)$ & $4.8(6)$ \\
Most of the time & $18.3(47)$ & $26.3(35)$ & $9.7(12)$ \\
Occasionally & $34.6(89)$ & $33.1(44)$ & $36.3(45)$ \\
Infrequently & $15.2(39)$ & $6.8(9)$ & $24.2(30)$ \\
Never & $22.2(57)$ & $19.5(26)$ & $25.0(31)$ \\
\hline
\end{tabular}

or 'most of the time' with others (in person), compared with only $14.5 \%$ of women $\left(n=124 ; \chi^{2}=29.494 ; P<0.001\right)$. Likewise, no female respondents indicated that they played digital games online 'every time', only two individuals $(1.6 \%$ of the 124 female respondents to this question) indicated that they played games online with others 'most of the time', and only two (1.6\%) suggested that they would play with others online 'occasionally'. This compares with $9.9 \%$ of male respondents (13 individuals) who stated that they played games with others online either 'every time', 'most of the time' or 'occasionally' - though this finding was not statistically significant $(P>0.05)$.

Significantly, respondents who played sport related digital games were also more likely than those who had not, to play digital games with others 'in person'. Of those who indicated that they had recently played a sport related digital game $(n=167), 39 \%$ suggested that they would play digital games with others in person either 'every time' or 'most of the time', compared with only $8 \%$ of those who had not recently played a sport game $\left(\chi^{2}=49.420 ; P<0.001\right)$. This, to a large degree, can be explained by sport related digital games frequently allowing more than one player to play simultaneously, but once again, emphasises the potential sociability of digital gaming, and particularly sport related games.

Overall, by far the most popular type of game for respondents to this survey were 'sport related games' with 38\% selecting this as their preferred type of game. However, these patterns were again very gendered. Sport related games were by far the most popular type of game for male respondents, with $52.4 \%$ selecting these, far ahead of second placed 'action' games (17.1\%) and 'strategy' games

Table 2 Percentages of how frequently respondents play digital games with other people via the Internet (number) $(n=257)$

\begin{tabular}{lccc}
\hline Play with others 'online' & Overall & Male & Female \\
\hline Every time & $0.4(1)$ & $0.8(1)$ & $0.0(0)$ \\
Most of the time & $2.7(7)$ & $3.8(5)$ & $1.6(2)$ \\
Occasionally & $3.5(9)$ & $5.3(7)$ & $1.6(2)$ \\
Infrequently & $11.7(30)$ & $14.3(19)$ & $8.9(11)$ \\
Never & $81.7(210)$ & $75.9(101)$ & $87.9(109)$ \\
\hline
\end{tabular}


Table 3 Percentages of respondents' favourite types of digital game (number) $(n=163)$

\begin{tabular}{lccc}
\hline Type of game & Overall & Men & Female \\
\hline Sport & $38.0(62)$ & $88.7(52.4)$ & $12.1(7)$ \\
Action (e.g. shoot 'em ups) & $14.7(24)$ & $17.1(18)$ & $10.3(6)$ \\
Strategy/war gaming/'god' simulations & $11.0(18)$ & $11.4(12)$ & $10.3(6)$ \\
Platform & $9.2(15)$ & $2.9(3)$ & $20.7(12)$ \\
Adventure/RPG & $8.6(14)$ & $7.6(8)$ & $10.3(6)$ \\
Driving/racing & $6.7(11)$ & $1.9(2)$ & $15.5(9)$ \\
Fighting (e.g. beat 'em ups) & $6.7(11)$ & $5.7(6)$ & $8.6(5)$ \\
Other & $4.9(8)$ & $1.0(1)$ & $12.1(7)$ \\
\hline
\end{tabular}

(11.2\%). In contrast, 'sport related games' were placed (joint) third by female respondents (with 12.1\%), behind 'driving/racing' (15.5\%) and 'platform' (20.7\%) games.

This gender divide was also noted in the number of male and female respondents who indicated that they had played a sport related digital game 'in the last three months'. Of those respondents who had recently played a digital game, $90.2 \%$ of men $(n=133)$ indicated that they had recently played a sport related game, compared with only $37.9 \%$ of women $\left(n=124 ; \chi^{2}=77.201 ; P<0.001\right)$. These figures are likely to be explained by the propensity for sport related games to be based almost exclusively on male dominated sports, such as soccer, American football, ice hockey and basketball. Generally, these types of games proved popular with male respondents, but far less so for women. Of the female respondents who indicated that they had recently played a sport related digital game $(n=47)$, multi-sport games (e.g. track and field and winter sports), and less gender specific games or non-team based games (which often allow players to chose female characters), such as golf and skateboarding, proved more popular than with the male respondents.

Overall, the results gathered from this questionnaire indicated that women were a lot less likely than their male peers to play digital games, and also played these a lot less frequently. As indicated, digital games had recently been played by the vast majority of male respondents to the questionnaire, and around half of the female respondents. Furthermore, as Table 4 indicates, it is also evident that generally women would play these games a lot less frequently, with only 23.2\% (compared

Table 4 Percentages of how frequently respondents play digital games (number) $(n=254)$

\begin{tabular}{lccr}
\hline Frequency & Overall & Male & Female \\
\hline Most days & $22.0(56)$ & $34.6(46)$ & $8.3(10)$ \\
2 or 3 times a week & $23.6(60)$ & $31.6(42)$ & $14.9(18)$ \\
Once a week & $16.1(41)$ & $14.3(19)$ & $18.2(22)$ \\
2 or 3 times a month & $18.1(46)$ & $12.0(16)$ & $24.8(30)$ \\
Once a month & $9.4(24)$ & $5.3(7)$ & $14.0(17)$ \\
Less than once a month & $10.6(27)$ & $2.3(3)$ & $19.8(24)$
\end{tabular}


with $66.2 \%$ of men) indicating that they would play digital games at least 'two or three times as week'.

These findings, which suggest that female respondents to this sample are considerably less likely to play digital games than their male counterparts, seems to go against the growing body of literature that suggests that young women are now almost as likely as their male peers to play digital games. Although this research differs methodologically to previous studies (such as Funk, 1993; Littleton et al., 1998; Colwell and Payne, 2000), its results indicate significantly greater variations in levels of digital game play between male and female respondents. It is likely that the variation in findings from this research, and those prior to this, may largely be explained by age and social situation differences between the sample groups. All of the respondents in this sample were over the age of 18 , and the vast majority were university students living away from home.

Haddon (1992) indicates that there appears to be little gender differences in boys' or girls' liking of playing digital games, and certainly the follow up interviews conducted for this research indicate that many women at least had the desire to play digital games. For instance all but one of the female interviewees suggested that they enjoyed playing digital games, though only one of these played frequently. The comments made by 'Shelly' (female, aged 21) were typical:

Yeah, I do really like playing games like on the computer... when I get chance, like when I'm round friends and stuff or when I'm back home with me brother...but that's not really that often, 'cos like I said, I don't have a games machine myself or nothing.

It has been suggested that the vast majority of digital gaming takes place in a domestic setting (i.e. in the home), often replacing the role of the traditional board games for many families (Squire, 2002). However, this research conducted on university students appears to suggest that away from the family home, women are a lot less likely than their male peers to continue gaming, and this is particularly noted in relation to sport related games. Haddon (1992) suggests that digital gaming can frequently constitute a male subculture. Similarly elsewhere (Crawford and Gosling, 2004), I have argued that men frequently restrict access for women to sport fan cultures. Hence, in relation to sport related digital games, it appears that many women may be doubly excluded. However, the increased popularity of certain male dominated sports (such as soccer) with many women (particularly in the US), has led Cassell and Jenkins (2000) to suggest that women may constitute a fresh and new market for sport related games developers in the future.

\section{Conclusion}

The initial aim of this research was to consider the relationship between sporting and digital gaming interests. In support of Fromme (2003), this data set provides no evidence to suggest that participation in digital gaming can have a negative affect on patterns of sport participation. However, as with Fromme, this data set is too small to provide statistically significant data, and consequently, further and more detailed research is required. However, what this research has indicated is that for many, sport related digital gaming can prove a popular source of 
conversation, cross-cutting and informing general conversations on sport. Digital gaming can inform and increase both interest and knowledge of sport, and in turn, interest in sport both as a participant and follower, can encourage some to play digital versions of these sports. It appears that, for many digital game players, there is a strong link and relationship in their social interactions and interests based around sport and the digital versions of these. These data therefore challenge the view that digital gaming practices are either inherently anti-social or deter individuals form participating in sport, as there appears a notable (and to some degree reciprocal) relationship between sporting and digital gaming interests for respondents in this sample group.

However, this research provides a relatively small sample, and more extensive research is needed into the relationship between sport and digital gaming. Moreover, this research reinforces the need to consider digital gaming in its social and cultural context (Yates and Littleton, 2001; Squire, 2002). For too long research on digital gaming has been based largely upon the interaction and relationship between the gamer and the games they play, such as psychological studies on increased levels of aggression in gamers (see Colwell and Payne, 2000). However, there needs to be an increased awareness and consideration of the location of these within their social context, and specifically a more detailed empirical understanding of how gaming is located within people's everyday lives and social networks.

There also needs to be further research into the continued importance of gender in shaping interest and levels of participation in digital gaming. As already stated, it was not the original intention of this research to seek out and identify gender differences. However, the data provided some striking differences between male and female respondents. In particular, this research indicates that women were significantly less likely to play digital games than their male counterparts, and this was particularly noted in relation to sport related games. Significantly, these findings vary greatly from many previous studies, such as Funk (1993) and Colwell and Payne (2000), which suggest that women are now almost as likely as men to play digital games. The variation in findings from this study, compared with previous research, is most likely explained by differences in the age and social situation of the samples. Where a large proportion of previous research has focused almost exclusively on school children, this survey samples adult gamers, and suggests that outside of a domestic context (i.e. the family home) women remain less likely than their male peers to play digital games.

\section{Notes}

1. Though terms are often used interchangeably, the term 'video games' is often used to refer to games played on home-based game consoles or on arcade machines, while 'computer games' are generally defined as those played on PC or Apple Macintosh systems (see Poole, 2000: 35). However, to help avoid confusion, the term 'digital gaming' appears to be the preferred term growing in acceptance within the literature on gaming to refer to all forms of electronic gaming, including video, computer and mobile gaming (see DiGRA).

2. The questionnaire had asked respondents who had recently ('in the last month') played sport to indicate how frequently they played these on a scale of 'most days', 2 or 3 times a week', 'once a week', 2 or 3 times a month', 'once a month' and 'less than once a month'. 
3. The questionnaire had asked respondents who had recently ('in the last three months') played a digital game to indicate how frequently they played these on a scale of 'most days', 2 or 3 times a week', 'once a week', 2 or 3 times a month', 'once a month' and 'less than once a month'.

\section{References}

Buchanan, D. D. and Funk, J. B., (1996) Video and computer games in the 90s: children's time commitment and games preferences, Children Today, 24, pp. 12-15.

Bryce, J. and Rutter, J. (2001) In the game - in the flow: presence in public computer gaming, Poster presented at Computer Games and Digital Textualities, IT University of Copenhagen, March 2001, available at http:// www.digiplay.org.uk (accessed 31 March 2003).

Bryce, J. and Rutter, J. (2002) Killing like a girl: gendered gaming and girl gamer's visibility, Presented at Computer Games and Digital Cultures, Tampere, Finland, June 2002.

Bryce, J. and Rutter, J. (2003a) The gendering of computer gaming: experiences and space, in: S. Fleming and I. Jones Leisure Cultures: Investigations in Sport, Media and Technology, pp. 3-22 (Eastbourne: Leisure Studies Association).

Bryce, J. and Rutter, J. (2003b) Gender dynamics and the social and spatial organization of computer gaming, Leisure Studies, 22, 1-15.

Cassell, J. and Jenkins, H. (2000) Chess for girls: feminisms and computer games, in: J. Cassell and H. Jenkins From Barbie to Mortal Combat: Gender and Computer Games, pp. 2-45 (London: MIT Press).

Colwell, J., Grady, C. and Rhaiti, S. (1995) Computer games, self-esteem and gratification of needs in adolescents, Journal of Communication and Applied Social Psychology, 5(3), pp. 195-206.

Colwell, J. and Payne, J. (2000) Negative correlates of computer game play in adolescents, British Journal of Psychology, 91, pp. 295-310.

Crawford, G. and Gosling, V. K. (2004) The myth of the puck bunny: female fans and men's ice hockey, Sociology, 38(3), pp. 477-493.

Deem, R. (1982) Women, leisure and inequality, Leisure Studies, 1, pp. 29-46.

Deem, R. (1986) All Work and No Play? (Milton Keynes: Open University Press).

Dietz, T. L. (1998) An examination of violence and gender role portrayals in video games: implications for gender socialization and aggressive behaviour, Sex Roles, 38(5-6), pp. 426-442.

DiGRA (no date) Digital Games Research Association, http://www.digra.org.

ELSPA (2003) The Cultural Life of Computer and Video Games: A Cross Industry Study. Entertainment and Leisure Software Publishers Association, available at http://www.elspa.com.

Funk, J. B. (1993) Re-evaluating the impact of computer games, Clinical Pediatrics, 32, pp. 86-90.

Fromme, J. (2003) Computer games as a part of children's culture, Game Studies, 3(1), available at http:// www.gamestudies.org/0301/fromme/.

Greenfield, P. M. (1996) Video games as cultural artifacts. (Special issue: Effects of interactive environment technologies on development) Journal of Applied Developmental Psychology, 15, pp. 3-12.

Haddon, L. (1992) Explaining ICT consumption: the case of the home computer, in: R. Silverstone and E. Hirsch Consuming Technologies: Media and Information in Domestic Spaces, pp. 82-96 (London: Routledge).

IDSA (2002) Essential Facts about the Computer and Video Game Industry, Interactive Digital Software Association, available at http://www.idsa.com/IDSABooklet.pdf.

Kafai, Y. B. (1996) Electronic play worlds: gender differences in children's construction of video games, in: Y. B. Kafai and M. Resnick Constructionism in Practice: Designing, Thinking and Learning in a Digital World, pp. 97-111 (Mahwah, NJ: Ablex).

Kielser, S., Sproull, L. and Eccles, J. (1985) Poolhalls, chips and war games: women in the culture of computing, Psychology of Women Quarterly, 4, pp. 451-462.

Kinder, M. (1991) Playing with Power in Movies, Television and Video Games: From Muppet Babies to Teenage Mutant Ninja Turtles (Berkley: University of Berkley Press).

Littleton, K., Light, P., Joiner, R., Messer, D. and Barnes, P. (1998) Gender, task scenarios and children's computer-based problem solving, Educational Psychology, 18(3), pp. 327-340.

O'Connor, A. (2002) Even better than the real thing? The Times (The Game suppl.), 9 December 2002, pp. 2-3.

Poole, S. (2000) Trigger Happy: The Inner Life of Videogames (London: Fourth Estate).

Roe, K. and Muijus, D. (1998) Children and computer games, European Journal of Communication, 13(2), pp. 181-200. 
Shaw, S. M. (1994) Gender, leisure and constraint: towards a framework for analysis of women's leisure, Journal of Leisure Research, 26, pp. 8-22.

Squire, K. (2002) Cultural framing of computer/video games, Game Studies 2(1), available at http://www.gamestudies.org/0201/squire/.

Wearing, B. (1998) Leisure and Feminist Theory (London: Sage).

Yates, S. J. and Littleton, K. L. (2001) Understanding computer game culture: a situated approach, in: E. Green and A. Adams Virtual Gender: Technology, Consumption and Identity, pp. 103-123 (London: Routledge). 